EPJ Web of Conferences 59, 17013 (2013)

DOI: $10.1051 /$ epjconf/20135917013

(C) Owned by the authors, published by EDP Sciences, 2013

\title{
Laser-plasma booster for ion post acceleration
}

\author{
D. Satoh ${ }^{1, a}$, S. Kawata ${ }^{1}$, K. Takahashi ${ }^{1}$, T. Izumiyama ${ }^{1}$, D. Barada ${ }^{1}$, Y.Y. Ma ${ }^{2}$, \\ Q. Kong ${ }^{3}$, P.X. Wang ${ }^{3}$, W.M. Wang ${ }^{1,4}$, Y.T. Li ${ }^{4}$, Z.M. Sheng ${ }^{4,5}$, O. Klimo ${ }^{6}$, \\ J. Limpouch ${ }^{6}$ and A.A. Andreev ${ }^{7}$
}

1 Utsunomiya University, Utsunomiya 321-8585, Japan

2 National University of Defense Technology, Changsha 410073, China

${ }^{3}$ Fudan University, Shanghai 200433, China

4 Inst. of Physics, CAS, Beijing 100190, China

5 Shanghai Jiao Tong University, Shanghai 200240, China

${ }^{6}$ Czech Technical University, CZ-182 121 Praha 8, Czech Republic

7 Inst. of Laser Physics, St. Petersburg 199034, Russia

\begin{abstract}
A remarkable ion energy increase is demonstrated for post acceleration by a laser-plasma booster. An intense short-pulse laser generates a strong current by high-energy electrons accelerated, when this intense short-pulse laser illuminates a plasma target. The strong electric current creates a strong magnetic field along the high-energy electron current in plasma. During the increase phase in the magnetic field, a longitudinal inductive electric field is induced for the forward ion acceleration by the Faraday law. Our 2.5dimensional particle-in-cell simulations demonstrate a remarkable increase in ion energy by several tens of $\mathrm{MeV}$.
\end{abstract}

\section{INTRODUCTION}

By chirped pulse amplification, high laser intensities have been realized, and high intensity short pulse lasers are now available for experiments and applications. On the other hand, ion beams are useful for basic particle physics, medical ion therapy, controlled nuclear fusion, high-energy sources, and so on. The energy of ions, which are accelerated in an interaction between an intense laser pulse and a near-critical density target, are over a few tens of $\mathrm{MeV}$ [1-6]. The issues in laser ion acceleration include ion beam collimation, ion energy spectrum control, ion production efficiency, etc. [1-28]. Depending on ion beam applications, the ion particle energy should be controlled. For example, ion beam cancer therapy needs $100 \sim 150 \mathrm{MeV}$ for proton energy. Therefore, in this paper we focus on a boost of ion beam energy by post-acceleration in laser plasma interaction. In this paper we propose a laser-plasma booster as an ion post acceleration scheme. In our study, we employ an intense shortpulse laser and a near-critical density plasma target, which consists of hydrogen. Figures 1(a) and (b) show the conceptual diagram of the laser-plasma booster. Figure 1(a) presents just a possible example scheme for a future multi-stage laser accelerator. In this paper we focus on one post acceleration stage. In this paper, we perform 2.5-dimensional particle-in-cell simulations to investigate the ion beam post-acceleration.

\footnotetext{
ae-mail: mt106639@cc.utsunomiya-u.ac.jp
}

This is an Open Access article distributed under the terms of the Creative Commons Attribution License 2.0, which permits unrestricted use, distribution, and reproduction in any medium, provided the original work is properly cited. 
EPJ Web of Conferences

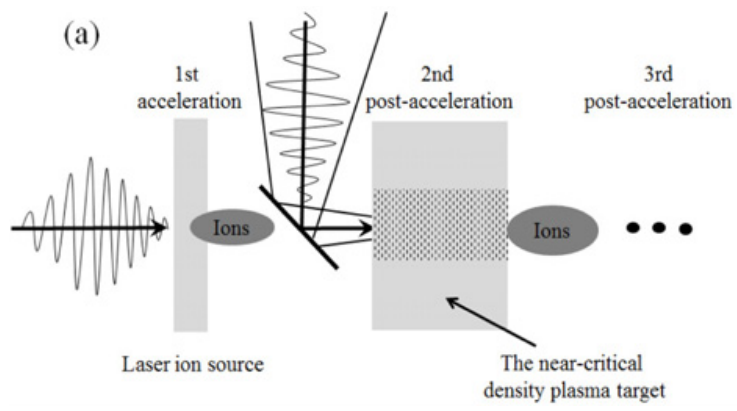

(b)

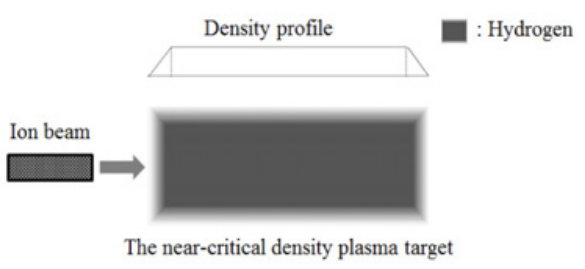

Figure 1. (a) An example concept for future ion post acceleration scheme and (b) the near-critical density hydrogen plasma target. The near-critical density plasma booster target provides a stable inductive acceleration field, for post acceleration of laser produced ions.

\section{LASER-PLASMA BOOSTER FOR ION POST ACCELERATION}

\subsection{The near-critical density plasma target simulation}

We perform 2.5-dimensional particle-in-cell simulations. The target model is shown in Fig. 1(b). The near-critical density plasma target is located in $9.5 \lambda<x<49.5 \lambda$ and $19.0 \lambda<y<31.0 \lambda$. The plasma target has a flat-top density profile. The edge region has a linear density gradient from $0 n_{c}$ to the maximum density of $0.5 n_{c}$, and has a linear density gradient in $2 \lambda$ in the $X$ and $Y$ directions at the target edges. The laser intensity is $I=1.0 \times 10^{20} \mathrm{~W} / \mathrm{cm}^{2}$, the laser spot diameter is $4.0 \lambda$, and the pulse duration is $40 \mathrm{fs}$. The laser transverse profile is Gaussian, and the laser temporal profile is also Gaussian. The laser wavelength is $\lambda=1.053 \mu \mathrm{m}$. The simulation box is $80 \lambda$ in the longitudinal direction and $30 \lambda$ in the transverse direction. The near-critical density plasma target density is $0.5 n_{c}$ and the ion beam density is $10^{16} \mathrm{~cm}^{-3}$. The pre-accelerated proton beam has the initial mean energy of $110 \mathrm{MeV}$, has the initial proton beam temperature of $2 \mathrm{MeV}$ and is located at the left of the plasma target initially. The initial proton beam size is $2 \lambda$ in $X$ and $5 \lambda$ in $Y$. The size and the initial temperature depend on the pre-acceleration mechanism. The size of the pre-accelerated ion beam and its mean energy are selected here to demonstrate the post-acceleration mechanism by the inductive acceleration.

\subsection{Post-acceleration of the ion beam}

Figures 2 show the distributions of acceleration electric field $E_{x}$ in $\mathrm{MV} / \mu \mathrm{m}$ at (a) $t=110 \mathrm{fs}$ and (b) $t=230 \mathrm{fs}$ and the distributions of magnetic field $B_{z}$ in Tesla at (c) $t=110 \mathrm{fs}$ and (d) $t=230 \mathrm{fs}$. The laser generates the high-energy electrons inside of the target. A magnetic field is also formed along the channel in the laser plasma interaction [26-28]. When the intense laser pulse propagates through the plasma, it accelerates a part of electrons. The electrons form a high current and generate the magnetic field. In the laser plasma interaction, the ion dynamics is affected directly by the electric field and the behavior of the electrons. The electrons form a strong magnetic field, and during the increase in the azimuthal magnetic field a strong inductive electric field is generated [6]. The ions are accelerated by the inductive electric field. The inductive acceleration field moves with a speed less than c, depending on the plasma density. At this target density of $0.5 n_{c}$, the speed of the inductive electric field is about $0.66 \mathrm{c}$. Therefore, the inductive acceleration field is appropriate for ion post acceleration and is rather stable. Figure 3 shows the ion beam energy distribution at $t=50 \mathrm{fs}, 230 \mathrm{fs}$ and $450 \mathrm{fs}$ for the nearcritical density plasma target. At $t=50 \mathrm{fs}$, Fig. 3 shows the initial energy of the ion beam which is not yet accelerated. The maximum ion beam energy reaches $171.2 \mathrm{MeV}$ from the initial energy of $110 \mathrm{MeV}$. The energy conversion efficiency from laser to the pre-accelerated protons is $4.67 \times 10^{-2} \%$, and the 

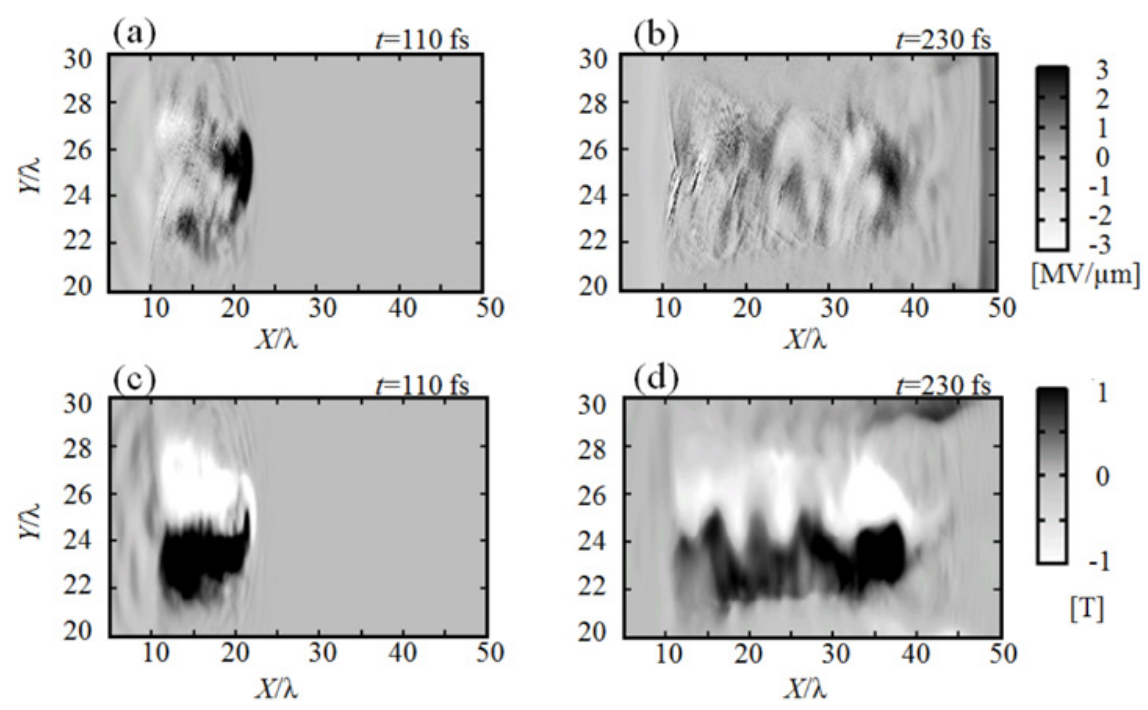

Figure 2. The distributions of acceleration electric field $E_{x}$ in $M V / \mu \mathrm{m}$ at (a) $t=110 \mathrm{fs}$ and (b) $t=230 \mathrm{fs}$, and the distributions of magnetic field $B_{z}$ in Tesla at (c) $t=110 \mathrm{fs}$ and (d) $t=230 \mathrm{fs}$.

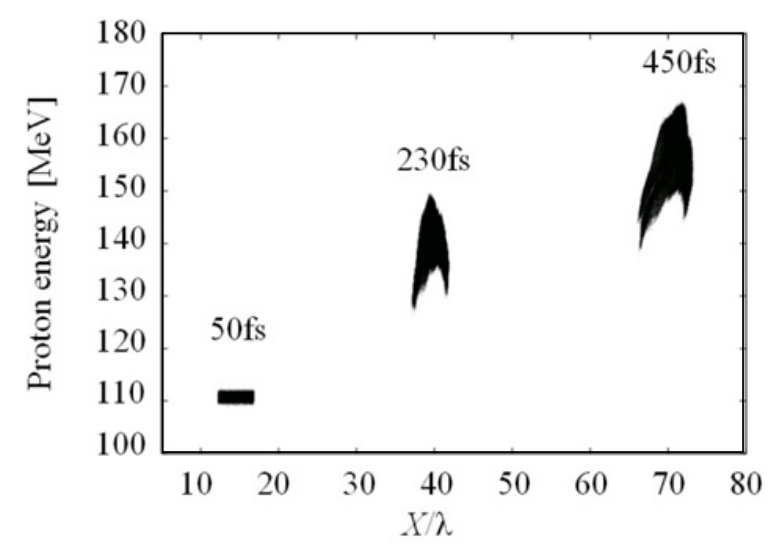

Figure 3. The ion beam energy distributions at $t=50 \mathrm{fs}, 230 \mathrm{fs}$ and $450 \mathrm{fs}$ for the near-critical density plasma target. At $t=50 \mathrm{fs}$, the ion beam is not yet accelerated.

energy efficiency to the background target protons is $35.5 \%$. The energy coupling efficiency to the preaccelerated proton beam is rather low. However, the present result shows a possible post-acceleration mechanism with successive inductive accelerations. Figure 3 shows that the final energy spread of protons becomes large compared with the initial energy spread. The final energy spread influences the beam quality or particle selection device parameters depending on ion beam utilization purposes. The requirement of the final beam quality should be addressed in the future, when the application purposes are specified.

\section{CONCLUSIONS}

In this paper we have proposed a laser-plasma booster as an ion post-acceleration scheme. We succeeded to increase the energy of the ion beam by the inductive acceleration field in a near-critical density 
plasma target illuminated by an intense short-pulse laser. The post-accelerated ion maximum energy increases from $110 \mathrm{MeV}$ to $171.2 \mathrm{MeV}$ by the strong inductive acceleration electric field. The work in this paper presents an important method for ion energy control in laser plasma acceleration. For practical applications of the laser ion accelerator the issues include the post-acceleration of the ion beam as studied in this paper, the ion beam quality improvement in the energy spectrum control including a mono-energetic ion beam generation, neutralized or unneutralized ion beam transportation for a long distance, etc. This study presents a new way to generate and to control the ion beam energy.

This work is partly supported by MEXT, JSPS ILE/Osaka Univ. and CORE (Center for Optical Research and Education, Utsunomiya Univ., Japan). J.L. and O.K. gratefully acknowledge partial support by Czech Science Foundation (P205/11/1165) and Czech Ministry of Education (MSM6840770022).

\section{References}

[1] K. Takahashi et al., Phys. Plasmas 10, 093102 (2010)

[2] M. Nakamura et al., J. Appl. Phys. 101, 113305 (2007)

[3] Y. Nodera and S. Kawata, Phys. Rev. E 78, 046401 (2008)

[4] I. Velchev, E. Fourkal, and C. M. Ma, Phys. Plasmas 14, 033106 (2007)

[5] S. V. Bulanov et al., Plasma Phys. Rep. 30, 18 (2004)

[6] T. Nakamura, S. Kawata, Phy. Rev. E 67, 026403 (2003)

[7] Y. Y. Ma et al., Phys. Plasmas 13, 110702 (2006)

[8] R. Sonobe et al., Phys. Plasmas 12, 073104 (2005)

[9] F. De Lillo, F. Cecconi, G. Lacorata, A. Vulpiani, Eur. Phys. Lett. 84 (2008)

[10] A. P. Robinson, A. R. Bell, and R. J. Kingham, Phys. Rev. Lett. 96, 035005 (2006)

[11] M. Roth et al., Phys. Rev. Lett. 86, 436 (2001)

[12] M. Kaluza et al., Phys Rev. Lett. 93, 045003 (2004)

[13] Y. Sentoku et al., Phys. Rev. E 62, 7271 (2000)

[14] S. Ter-Avetisyan et al., Phys. Rev. Lett. 96, 145006 (2006)

[15] A. A. Andreev, K. Yu. Platonov, T. Okada and S. Toraya, Phys. Plasmas 10, 220 (2003)

[16] A. A. Andreev et al., Plasma Phys. Control. Fusion 48, 1605 (2006)

[17] A. A. Andreev and J. Limpouch, J. Plasma Phys 62, 179 (1999)

[18] A. A. Andreev et al., Laser and Particle Beams 22, 431 (2004)

[19] T. P. Yu et al., Phys. Plasmas 16, 033112, (2009)

[20] E. Fourkal, I. Velchev and C. M. Ma, Phys. Rev. E 71, 036412 (2005)

[21] S. P. Hatchett et al., Phys. Plasmas 7, 2076 (2000)

[22] M. Borghesi et al., Phys. Rev. Lett. 92, 055003 (2004)

[23] F. Lindau et al., Phys. Rev. Lett. 95, 175002 (2005)

[24] T. Zh. Esirkepov et al., Phys. Rev. Lett. 89, 175003 (2002)

[25] T. Zh. Esirkepov et al., Phy. Rev. Lett. 92, 175003 (2004)

[26] T. Nakamura et al., Phys. Rev. Lett. 105, 135002 (2010)

[27] Y. Fukuda et al., Phys. Rev. Lett. 103, 165002 (2009)

[28] S. V. Bulanov and V. S. Khoroshkov, Plasma Phys. Rep. 28, 453 (2004) 